\title{
EDITORIALS
}

\section{Moving Medical Advances from Prototype into Practice}

\author{
Bruce L. Rollman, MD, MPH \\ Division of General Internal Medicine, Center for Research on Health Care, University of Pittsburgh School of Medicine, Pittsburgh, PA, USA.
}

$\mathrm{J}$ Gen Intern Med 25(7):640-1

DOI: $10.1007 / \mathrm{s} 11606-010-1355-4$

(c) Society of General Internal Medicine 2010

I mproving health care outcomes is difficult, and we have known for many years that successful efforts are more likely to involve a blend of approaches rather than a single "silver bullet." ${ }^{1}$ Over the past 15 years, dozens of published trials have supported use of the multipronged "collaborative care" model as an effective strategy for improving treatment outcomes for depression $^{2-4}$ as well as for a variety of other chronic medical conditions. ${ }^{5}$ Based on the Wagner Chronic Care Model, collaborative care involves active follow-up by a non-physician allied health professional "care manager" who supports patients with the time and frequency of contacts necessary to educate them about their illness and evidence-based treatment options; involves primary care physicians as active participants in their patients' care; and proactively monitors patients' response to therapy and suggests adjustments in care when indicated. Yet despite the proven efficacy and cost-effectiveness of this approach, collaborative care strategies have yet to be implemented widely into routine practice beyond a few large integrated health care delivery organizations. ${ }^{6}$ This lack of implementation may be due to factors ranging from the complexity, effort, and costs associated with the change (e.g., electronic medical records); reluctance by health plans to pay for new treatments and modalities of care delivery (e.g., telehealth); development of novel treatments in isolation from the real world of patient care and attention towards financial profits and losses (e.g., unbillable care manager time); lack of demand for services, particularly by patients who may be stigmatized by their condition (depression); and investigator lack of follow-through or skill with the science of implementation. ${ }^{7}$

These implementation challenges are not unique to collaborative care. Routine acceptance of beta-blockers, statins, and aspirin following myocardial infarction may take 15 years or more from publication of the major trial demonstrating efficacy to achieve high usage rates in clinical practice. ${ }^{8}$ Compare this with a story related to me by a colleague who has a friend in Hollywood: major film studios typically devote one-third of a movie's budget to production and the balance to marketing and distribution. A film must also have a distribution plan before given the "green light" on production. Moreover, compensation of Hollywood producers and directors is based not on their film's critical acclaim (production), but rather on its box office receipts and profits (dissemination and implementation). By contrast, approximately $98 \%$ of the cost of the typical research grant goes for "production" and $2 \%$ is devoted to "distribution,"

Published online April 23, 2010 mostly in the form of the traditional academic products of scientific presentations and publications that are valued by university promotion committees.

The National Institutes of Health (NIH) spends billions of dollars annually on biomedical research. In response to public concerns about the practical return on its investment, the NIH developed the Roadmap for Medical Research to speed the translation of basic science research into clinical practice. ${ }^{9}$ The Roadmap centers on two translation steps: from basic science to intervention development and testing (T1); and from intervention development to implementation in clinical practice (T2). Yet, compared to the resources devoted to basic science, scant resources are devoted to test how best to disseminate strategies that can potentially implement proven effective advances into the community settings where most patients obtain care (T3). ${ }^{10}$ Furthermore, the NIH does not fund efforts to convert "prototype" models of care developed under grant-supported conditions into sustainable interventions that can be delivered under real-world conditions, and few investigators have successfully overcome this barrier. ${ }^{11}$

Closing the gap between research discovery and program delivery is both a complex challenge and an absolute necessity if the U.S. public is to benefit from its investment in new scientific discoveries. In recent years, the NIH and other Federal agencies have devoted increased attention to these issues. For example, the Third Annual NIH Conference on the Science of Dissemination and Implementation, held in Bethesda, Maryland this past March, was a forum to exchange ideas about how best to apply knowledge of proven-effective treatments so as to inform policy and practice and expand the pertinent evidence base. Clinicianinvestigator readers of JGIM may benefit by browsing the conference website ${ }^{12}$ and several recent NIH program announcements so as to keep their own work relevant in these times of changing research priorities (e.g., PAR-10-038 and PAR-10-040).

New opportunities to close the translational gap are also emerging. As with passage of Medicare Part $\mathrm{D},{ }^{13}$ recently passed legislation to overhaul the U.S. health system will provide investigators opportunities and resources to produce time-sensitive research about the impact of these changes. These critical studies will inform policymakers of possible adjustments to new healthcare regulations. Critically, the new health care law will support a center for innovation within the Department of Health and Human Services to test and evaluate ways of improving quality and reducing the cost of health care. These pilot programs are likely to include novel strategies to reorganize and reimburse primary care physicians for providing high-quality chronic illness care, of which collaborative care is likely to be an integral part. ${ }^{5,14}$

Pertinent to the issues of universal access, coordinated care, and increased usage of electronic medical record systems promoted by the Obama Administration are two reports in this issue of JGIM. Hooshyar et al.'s comparison of the time to initiation of depression treatment between HIV-infected and 
uninfected patients enrolled in the Veterans Aging Cohort Study (VACS) ${ }^{15}$ confirms our prior understanding that severely depressed patients are more likely to be recognized and treated for this condition than less severely affected individuals. It also extends our knowledge to an HIV-infected population receiving care within a single-payer system where clinicians make extensive use of a sophisticated electronic medical record system. Reassuringly, they report the severity of patients' mood symptoms, rather than their HIV-status, is the critical determinant of whether a depression treatment is offered. ${ }^{16}$ As screening, feedback, and initiation of depression treatment by primary care clinicians is generally insufficient at improving patient outcomes without an organized system of follow-up, ${ }^{17}$ we await a follow-up report from VACS on whether feedback of the depression diagnosis within VA settings that support chronic disease care managers actually improved patient outcomes (e.g., mood symptoms, CD-4 counts, viral loads, survival). Prins et al. conducted a secondary analysis of 721 Dutch patients with a current anxiety or depressive disorder recruited from 67 primary care practices. Despite their country's universal health care system and near $100 \%$ use of electronic medical record systems by general practitioners, ${ }^{18}$ under $40 \%$ of patients received guideline-concordant depression care, and their perceived need and accessibility to care were the greatest determinants to receipt of appropriate treatment. ${ }^{19}$ Thus we may conclude from these papers than patient factors are at least as important as system factors in determining delivery of appropriate depression care.

Medicine might never match Hollywood in its spending on product implementation. However, as we consider the best methods to improve treatment outcomes, the reports in this issue of JGIM remind us to look to a variety of health care delivery systems to inform U.S. policy as it undergoes substantial changes over the next few years. We look particularly forward to more reports from other countries that provide its citizens with universal access to primary care-centered systems of health care supported by electronic medical record system infrastructures for novel solutions to long-standing challenges. ${ }^{20}$ As a nation, we must advance towards the ultimate goal of accelerating adoption and integration of evidence and treatment strategies derived from prototype interventions into our routine daily practices and medical decision-making.

Corresponding Author: Bruce L. Rollman, MD, MPH; Division of General Internal Medicine, Center for Research on Health Care, University of Pittsburgh School of Medicine, Suite 600, 230 McKee Place, Pittsburgh, PA 15213-2582, USA (e-mail: rollmanbl@upmc. edu).

\section{REFERENCES}

1. Greco PJ, Eisenberg JM. Changing physicians' practices. N Engl J Med. 1993;329:1271-1274.

2. Gilbody S, Bower P, Fletcher J, Richards D, Sutton AJ. Collaborative care for depression: a cumulative meta-analysis and review of longer-term outcomes. Arch Intern Med. 2006;166:2314-2321.

3. Rollman BL, Belnap BH, LeMenager MS, et al. Telephone-delivered collaborative care for treating post-CABG depression: a randomized controlled trial. JAMA. 2009;302:2095-2103.

4. Katon WJ, Von Korff M, Lin EH, et al. The Pathways Study: a randomized trial of collaborative care in patients with diabetes and depression. Arch Gen Psychiatry. 2004;61:1042-1049.

5. Coleman K, Austin BT, Brach C, Wagner EH. Evidence on the chronic care model in the new millennium. Health Aff. 2009;28:7585

6. Shortell SM, Gillies R, Siddique J, et al. Improving chronic illness care: a longitudinal cohort analysis of large physician organizations. Med Care. 2009;47:932-939.

7. Proctor EK, Landsverk J, Aarons G, et al. Implementation research in mental health services: an emerging science with conceptual, methodological, and training challenges. Adm Policy Ment Health. 2009;36:24-34.

8. Lenfant C. Shattuck lecture-clinical research to clinical practice-lost in translation? N Eng J Med. 2003;349:868-874.

9. Zerhouni EA. US biomedical research: basic, translational, and clinical sciences. JAMA. 2005;294:1352-1358.

10. Westfall JM, Mold J, Fagnan L. Practice-based research-"Blue Highways" on the NIH roadmap. JAMA. 2007;297:403-406.

11. Blasinsky M, Goldman HH, Unutzer J, Blasinsky M, Goldman HH, Unutzer J. Project IMPACT: a report on barriers and facilitators to sustainability. Adm Policy Ment Health. 2006;33:718-729.

12. 3'rd Annual NIH Conference on the Science of Dissemination and Implementation; Bethesda, MD, March 15-16, 2010; http://conferences. thehillgroup.com/obssr/DI2010/index.html. Accessed March 22, 2010.

13. Zhang Y, Donohue JM, Lave JR, et al. The effect of Medicare Part D on drug and medical spending. N Engl J Med. 2009;361:52-61.

14. Iglehart JK. No place like home-testing a new model of care delivery. N Engl J Med. 2008;359:1200-1202.

15. Hooshyar D, Goulet J, Chwastiak L, et al. Time to depression treatment in primary care among HIV-infected and HIV-uninfected Veterans. J Gen Intern Med. 2010; doi:10.1007/s11606-010-1323-z.

16. Rollman BL, Hanusa BH, Gilbert T, Lowe HJ, Kapoor WN, Schulberg HC. The electronic medical record: a randomized trial of its impact on primary care physicians' initial management of major depression. Arch Intern Med. 2001;161:189-197.

17. Screening for depression in adults: U.S. preventive services task force recommendation statement. Ann Intern Med. 2009;151:784-792.

18. Wynand PMM, van de Ven Schut FT. Universal mandatory health insurance in the Netherlands: a model for the United States? Health Aff. 2008;27:771-781.

19. Prins MA, Verhaak PFM, Smolders M, et al. Patient factors associated with guideline-concordant treatment of anxiety and depression in primary care. J Gen Intern Med. 2010; doi:10.1007/s11606009-1216-1.

20. Computerized cognitive behavioral therapy for depression and anxiety. Review of Technology Appraisal 51. London: National Institute for Health and Clinical Excellence; February 2006. 